\title{
Las narrativas en torno a lo ibérico en los museos y yacimientos arqueológicos valencianos: de los discursos oficiales a las percepciones sociales
}

\author{
Narratives Around Iberian Culture at Valencia Museums and \\ Archaeological Sites: From Official Discourses to Social Perceptions
}

\author{
Tono Vizcaíno Estevan \\ Doctor en Arqueología por la Universitat de València \\ antonio.vizcaino@uv.es
}

Recibido: 07-03-2016

Aceptado: 05-07-2016

\begin{abstract}
RESUMEN
Los museos y los yacimientos arqueológicos constituyen los espacios más autorizados para hablar sobre el pasado y el patrimonio desde un punto de vista cientifico. Como tales, sus discursos son asumidos por los visitantes como veraces y rara vez son puestos en duda o contestados. Sin embargo, desde la arqueología debemos plantear una mirada crítica sobre las narrativas que se generan en estos espacios patrimoniales y el impacto que tienen sobre los visitantes, pues con frecuencia traducen y sancionan realidades politicas, sociales e identitarias que tienen más que ver con el presente que con el pasado. En este trabajo nos proponemos analizar los discursos oficiales sobre la cultura ibérica construidos en yacimientos y museos arqueológicos del País Valenciano, y contrastarlos con las percepciones sociales de los visitantes.
\end{abstract}

Palabras Clave: cultura ibérica, museos, yacimientos, imagen, percepciones sociales, País Valenciano.

\begin{abstract}
Museums and archaeological sites are considered the most authoritative places for talking about the past and the heritage from a scientific perspective. In fact, visitors assume their discourses as reliable and indisputable. In spite of that, professionals of archaeology must critically analyse the production of narratives at heritage sites, since they often reflect social, political and identity issues related to the present-day realities. The aim of this paper is to study official and popular discourses about the Iberian culture (Iron Age) collected in museums and archaeological sites from Valencia region.
\end{abstract}

KEYwords: Iberian Culture, Museums, Archaeological Sites, Image, Social Perceptions, Valencia Region.

SumARIO: 1. Introducción. 2. La imaginación del pasado como objeto de estudio. 3. Metodología de trabajo y muestra analizada. 4. Análisis de las narrativas en torno a lo ibérico. 5. Reflexiones finales. 


\section{Introducción}

En la actualidad son muchos y muy distintos los canales a través de los que la sociedad tiene la oportunidad de interactuar con el pasado. De entre todos ellos, los museos y los yacimientos arqueológicos ocupan un lugar prominente, no tanto por su capacidad de impacto cuantitativo, que es inferior a la de otros formatos como el televisivo (MECD 2015), sino por su reconocimiento social. En efecto, en ellos se dan dos circunstancias exclusivas que les permiten estar por encima del resto de formatos y asumir un valor simbólico de primera magnitud. Por un lado, constituyen los únicos lugares en los que se produce el contacto directo con la materialidad del pasado, de ahí su gran potencial evocador (Holtorf 2006). Por otro, son entendidos como los espacios más autorizados para explicar cómo sucedieron las cosas a lo largo de la historia, pues conjugan la prerrogativa de custodiar los restos arqueológicos con la cientificidad del conocimiento emanado de la Academia.

Esto los convierte en espacios de poder casi sacralizados (Gustafsson y Karlsson 2008; Hamilakis y Yalouri 1999) en los que domina un discurso experto y donde no hay cabida, en general, para otras maneras de entender el pasado que estén al margen de la oficialidad. Algo que no ocurre, por ejemplo, con las revistas y libros de divulgación, los cómics, las novelas históricas o los documentales. Así, difícilmente el visitante pone en entredicho lo que dicen museos y yacimientos, cuyos contenidos son asumidos como los más fidedignos por la mayoría. Este reconocimiento social, junto a las mejoras que se han producido en sus instalaciones y servicios para hacerlos más atractivos, ha convertido a muchos museos y yacimientos en un auténtico motor del turismo y del ocio cultural (Azuar 2013: 122). Además, por supuesto, de desempeñar una intensa labor educativa no solo en el conocimiento del pasado, sino también en el fomento de valores sociales y culturales.

Considerando la responsabilidad que asumen estos espacios patrimoniales, resulta pertinente preguntarse qué discursos se están construyendo sobre el pasado y hasta qué punto han calado en la sociedad. Por ello, en este texto nos planteamos como objetivo principal analizar las narrativas generadas tanto por los museos y los yacimientos como por la propia sociedad en torno a un pasado concreto, el ibérico, y en un territorio determinado, el País Valenciano.

\section{La imaginación del pasado como objeto de estudio}

Los estudios sobre la presentación del pasado prehistórico y protohistórico en yacimientos y museos arqueológicos no resultan extraños en el ámbito europeo (Collings 2007; Copeland 2004; Gustafsson y Karlsson 2008; Sommer 2008) y, aunque en menor medida, contamos con algunos ejemplos también en el Estado español (Ruiz Zapatero 1998 y 2013; Mansilla 2004; Masriera 2007).

El interés por este tipo de estudios parece responder mayoritariamente a la preocupación por la didáctica, así como a la importancia creciente de la divulgación en el marco del turismo y del consumo cultural, que han inducido a analizar y evaluar las posibilidades de estos espacios de aprendizaje. Pero, paralelamente, se han desarrollado estudios críticos que han cuestionado algunos de los estereotipos sociales, culturales y políticos que pueden rastrearse en los discursos de museos y yacimientos. En esta vía de investigación ha tenido especial incidencia el análisis de la cuestión de género (Porter 1990; Rísquez y Hornos 2000; Querol 2014; Hornos y Rísquez 2005; Querol y Hornos 2011 y 2015), pero también otras variables como las identidades étnicas y las relaciones de poder (Merriman 2000; Ballard 1997; Mouliou 2008; Smith 2000).

Menos frecuentes han sido los estudios sobre las percepciones en esos espacios patrimoniales. En este campo han predominado los trabajos encaminados a evaluar los recursos e instalaciones de yacimientos y, especialmente, de museos, impulsados por las propias instituciones para conocer a sus públicos (Pérez Santos 2008). A éstos se han añadido, en la última década, investigaciones sobre la percepción del patrimonio (Collings 2007; Ibáñez Alonso 2014; Masriera 2007; Merriman 2000; Scott 2005) y sobre sus significados sociales (Álvarez Vidaurre 2011; Holtorf 1997). En la mayoría de casos, desarrollados mediante la encuesta y la observación directa de los visitantes, si bien han cobrado protagonismo otros objetos de análisis, como los libros de visitas (Ayán 2015; Macdonald 2005; Rodríguez Temiño, Ruiz Cecilia y Mínguez García 2015).

\section{Metodología de trabajo y muestra analizada}

En este estudio hemos aplicado distintas metodologías para ofrecer una perspectiva lo más 


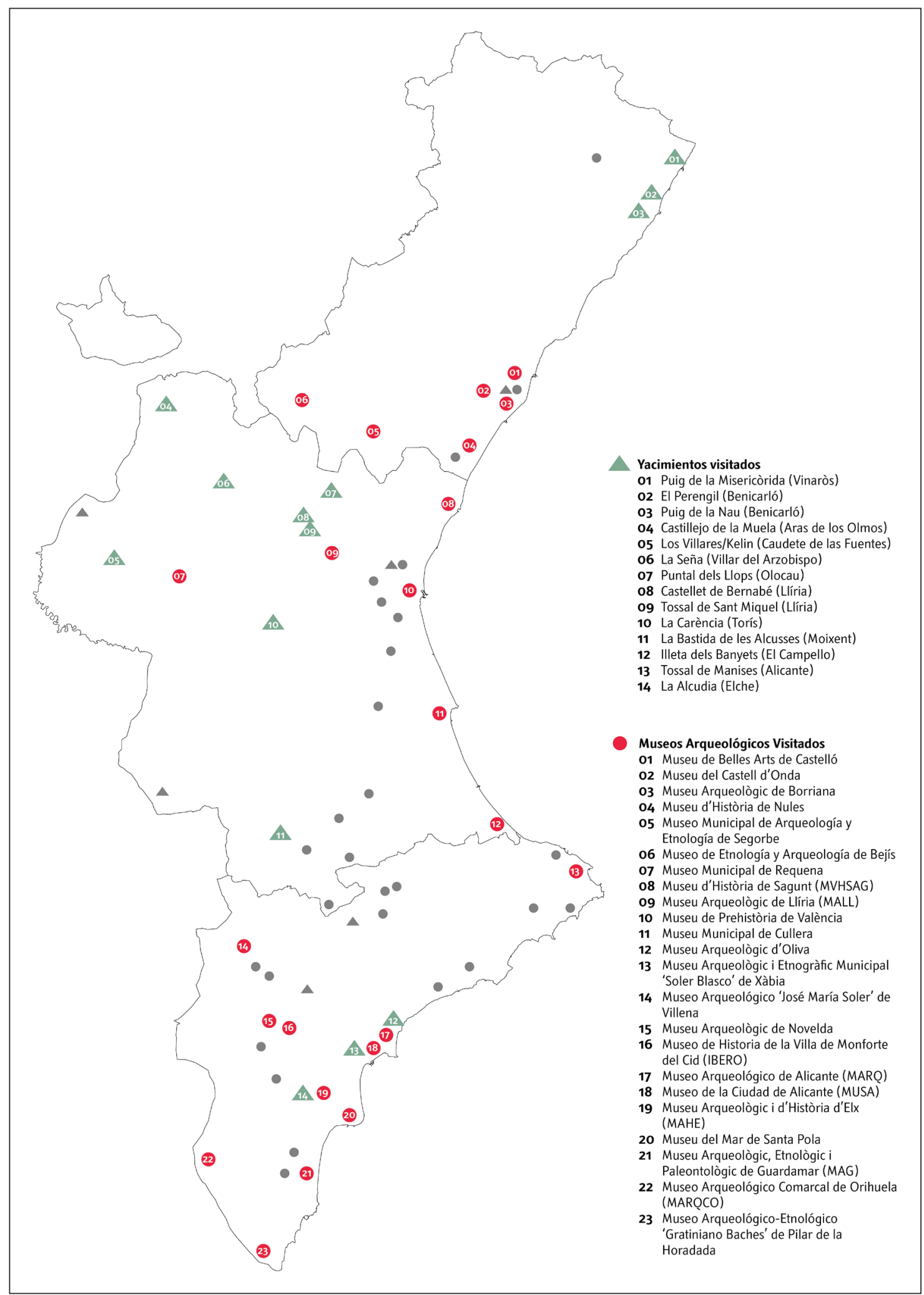

Figura 1. Museos y yacimientos arqueológicos que integran la muestra (Fuente:elaboración propia). 
completa posible. Para abordar los discursos de museos y yacimientos, hemos recurrido al análisis de contenido en dos direcciones distintas pero complementarias: las representaciones visuales, que constituyen un potente recurso para el anclaje de ideas sobre el pasado (Moser 2009), y los discursos textuales. Con el propósito de sistematizar la recogida de datos y su posterior estudio, hemos desarrollado un modelo de análisis y una serie de herramientas de trabajo (fichas y esquemas analíticos) aplicados a cada uno de los casos. En la selección de museos y yacimientos del conjunto del País Valenciano que integran la muestra (Fig. 1), han primado los siguientes criterios: disponer de un mínimo de información arqueológica (paneles, folletos), cuestión redundante para los museos pero no siempre contemplada en los yacimientos; garantizar la representatividad tanto del total de museos y yacimientos existentes como de su distribución territorial y poblacional; e incluir los distintos niveles de titularidad, gestión y proyección territorial.

Para el estudio de las percepciones, hemos aplicado métodos de investigación social de corte cuantitativo y cualitativo. Entre 2011 y 2013 desarrollamos un estudio sobre las percepciones del pasado ibérico en distintos yacimientos y museos valencianos, en el marco de una investigación de doctorado. De esos estudios se han introducido aquí algunas pinceladas. El trabajo de campo lo desarrollamos, por un lado, en las jornadas de puertas abiertas (JPA a partir de ahora) y recreación histórica de La Bastida de les Alcusses (Moixent) y de Los Villares-Kelin (Caudete de las Fuentes), donde aplicamos un total de 326 y 649 encuestas respectivamente. Por otro lado, en visitas y talleres en el Museu de Prehistòria de València, el Museo Arqueológico de Alicante (MARQ), El Tossal de Manises-Lucentum (Alicante) y L'Alcúdia de Elche, donde llevamos a cabo la observación participante.

\section{Análisis de las narrativas en torno a lo ibérico}

Entender cómo se construye y percibe el pasado ibérico en museos y yacimientos arqueológicos, requiere de un análisis global que va más allá del contenido de los recursos informativos (paneles, folletos, guías). En la imaginación del pasado influyen toda una serie de procesos cognitivos y emocionales que están condicionados por factores como el entorno inmediato, las facilidades de acceso, el uso de elementos simbólicos o el propio estado de conservación de los restos. No obstante, en este estudio dejaremos de lado los aspectos formales referidos a servicios, equipamientos y otros recursos relacionados con la accesibilidad, pues excede sus posibilidades, y nos centraremos en los contenidos que, directa o indirectamente, construyen las narrativas en torno a lo ibérico.

Partiendo de esta consideración, hemos estructurado el análisis del discurso en distintos apartados que se corresponden con aquellos elementos que, bajo nuestro punto de vista, tienen mayor incidencia en la percepción del pasado. El recorrido lo planteamos desde una perspectiva espacial, por lo que comenzaremos desde la escala superior -el territorio-, e iremos descendiendo hasta llegar al nivel más cercano, el de los recursos informativos del propio yacimiento. En cada apartado, y siempre que sea posible, ofreceremos un vis-à-vis, combinando las visiones oficiales con las percepciones de los visitantes.

\subsection{Ubicación y distribución en el territorio}

Los estudios sobre los espacios oficiales de divulgación del pasado, en especial los referidos a los yacimientos arqueológicos, suelen encontrar en el paisaje y el entorno inmediato la esfera máxima de análisis. Sin embargo, existe una escala superior que consideramos interesante tener en cuenta por la influencia que ejerce en la concepción de las culturas del pasado y, por su puesto, en la explicación de los procesos de patrimonialización. Nos referimos a la ubicación y distribución de los espacios patrimoniales en el territorio.

Esta variable resulta de gran interés cuando hablamos de yacimientos. En el País Valenciano se ha documentado un gran número de yacimientos ibéricos que se distribuyen a lo largo $\mathrm{y}$ ancho del territorio, aunque con niveles de densidad desiguales. Las diferencias son fruto de los propios patrones de ocupación en época ibérica, pero también de factores como la mayor o menor presión urbanística o el estado de las investigaciones arqueológicas en cada zona. Ahora bien, del conjunto de yacimientos ibéricos solamente unos pocos han sido acondicionados para la visita. Sin negar el peso que tiene en su selección la finalidad divulgativa y turística, que prima yacimientos en buen estado de conservación y con facilidades de acceso, 
es innegable que también influyen otros intereses, como los políticos y los identitarios. Así, la proliferación de yacimientos visitables de una época u otra habla de la importancia otorgada en cada territorio a las distintas épocas y culturas de su historia.

En el caso particular de la cultura ibérica en el País Valenciano, se constatan dos fenómenos reseñables. El primero de ellos es el peso cuantitativo de la provincia de Valencia, que aglutina 11 de los 20 yacimientos visitables. La mayoría de ellos se integran en la llamada Ruta dels Ibers, circunscrita exclusivamente al ámbito provincial, que ha sido impulsada por el Servei d'Investigació Prehistòrica (SIP) de la Diputació de València, en colaboración con los distintos ayuntamientos (Ferrer y Vives-Ferrándiz 2014). Frente al caso valenciano contrasta la escasez de Alicante, con cinco yacimientos accesibles, y especialmente Castellón, con tan sólo tres.

Estos desequilibrios son significativos en tanto en cuanto no responden necesariamente a una desigual riqueza arqueológica, sino más bien a políticas patrimoniales diferenciadas. Así, la mayor atención otorgada a los iberos en Valencia encaja con una tradición que defiende el territorio provincial valenciano, asociado a la antigua Edetania, como espacio privilegiado de la cultura ibérica. Dicha identificación fue impulsada entre finales del s. XIX y principios del s. XX por el movimiento cultural de la Renaixença, y encontró décadas más tarde en el SIP (1927) uno de sus principales elementos de legitimación, al apostar por un programa de excavaciones en el que lo ibérico asumía un claro protagonismo (Aranegui 2012: 43ss). Esa predilección por el pasado ibérico sigue estando muy presente hoy en día, y no afecta solo a las líneas de investigación desarrolladas por la institución, sino también a la divulgación, pues buena parte de sus actividades y de los proyectos de acondicionamiento de los restos -como la propia Ruta dels Ibers- está orientada a la difusión de la cultura ibérica.

El segundo de los fenómenos constatados en la distribución territorial de los yacimientos ibéricos visitables es la mayor concentración del interior frente a la costa. Aquí inciden distintos factores. En primer lugar, la destrucción sistemática de patrimonio en la llanura litoral debido a la intensa explotación industrial y agrícola, acelerada en las últimas décadas por el proceso de urbanización salvaje que ha afectado a la mayor parte de la costa valenciana. En segundo lugar, la primacía del turismo de sol y playa, que ha llevado a muchos municipios a marginar el patrimonio arqueológico. Aunque es cierto que la asociación dicotómica entre playa y turismo de ocio frente a interior y turismo cultural no es absoluta -y prueba de ello es Alicante y su territorio inmediato, con dos yacimientos de referencia (E1 Tossal de Manises-Lucentum y La Illeta dels Banyets) en una de las zonas turísticamente más masificadas del país- resulta innegable que la primacía del turismo de playa ha tenido efectos negativos sobre el patrimonio arqueológico. Así lo demuestra, por ejemplo, que centros turísticos clásicos como Benidorm, Torrevieja o Benicàssim carezcan de museos arqueológicos (Azuar 2013: 117). En tercer y último lugar, y en parte como consecuencia de lo anterior, las iniciativas impulsadas desde la administración para reactivar el interior a través de un turismo cultural sostenible y de calidad, tras décadas de abandono y despoblación (Bonet et al. 2007). Unas iniciativas que han encontrado en el patrimonio arqueológico un interesante aliciente para su desarrollo.

Lo cierto es que las asimetrías en la distribución territorial de los yacimientos ibéricos visitables influyen de manera directa en la concepción de la cultura ibérica. En concreto, asociándola a la zona interior, sobre todo de la provincia de Valencia, que es donde más yacimientos visitables existen. Cuando en las JPA preguntábamos por la distribución de la cultura ibérica en un mapa que incluía la España de las autonomías, el norte de África y el sur de Francia, entre el 5-10\% de los encuestados -dependiendo al año y el lugar de celebración- ubicaba a los iberos en zonas muy concretas de extensión comarcal o, a lo sumo, provincial. A pesar de no ser una de las categorías dominantes, y teniendo siempre presente que fueron realizadas en dos municipios del interior valenciano, dentro de ella la práctica totalidad de referencias ponían en relación a los iberos con puntos específicos del territorio valenciano. Unas veces distribuyéndolos por el interior de la provincia de Valencia; otras, amoldándolos al territorio exclusivamente provincial.

Pero lo interesante de este fenómeno no es solo el hecho de que, por concentración de yacimientos acondicionados para la visita, se llegue a establecer una relación directa entre los iberos y el interior valenciano; sino que, todavía más, ese territorio esté caracterizado por un paisaje que acaba siendo visto como definitorio de lo 
ibérico, conectando con algunas de las preconcepciones más habituales a la hora de imaginar esta cultura.

\subsection{El paisaje}

El paisaje, tanto el que envuelve a los yacimientos como el que se recrea en los museos, tiene una gran capacidad evocadora y es crucial para facilitar los viajes en el tiempo, que constituyen uno de los grandes atractivos del patrimonio arqueológico (Holtorf 2006; Lowenthal 1998). Un entorno que acompañe y donde no haya interferencias modernas evidentes da pie a una especie de viaje iniciático que permite situarse en el pasado (Ruiz Zapatero 2013: 12), a la vez que constituye un recurso de gran potencialidad didáctica para entender la conformación histórica del paisaje.

En la mayoría de ocasiones, no obstante, el paisaje actual dista de encajar con la realidad de la época representada, como resultado de las transformaciones que éste ha experimentado a lo largo del tiempo, sobre todo en el s. XX. Más que una fidelidad histórica, lo que favorecen los entornos boscosos y determinados paisajes agrícolas -muchas veces vistos como entornos ideales para los conjuntos arqueológicos- es la creación de una atmósfera que evoca un pasado idealizado como contrapunto a la industrialización y urbanización del presente. Se trata, en resumidas cuentas, de una manera de concebir la autenticidad. Así, la naturaleza es vista como una reserva inalterada del pasado (Santamarina 2008: 30), aún y cuando su aspecto actual sea resultado de procesos de transformación recientes que poco tienen que ver con el paisaje en época antigua. En cambio, en el patrimonio arqueológico se tiende a diferenciar entre lo original (los restos antiguos) y lo nuevo (las restauraciones y reconstrucciones).

En este sentido, es significativo que muy pocos de los yacimientos estudiados pongan en entredicho la correspondencia entre el paisaje actual y la época que se está explicando. Más bien al contrario, se aprovecha para favorecer la inmersión. Un entorno "salvaje", esto es, no urbanizado, es clave para una visita satisfactoria. De hecho, a menudo son los valores medioambientales los que motivan la visita a yacimientos arqueológicos (Sommer 2008: 167; Ayán 2015: 120). Una realidad que hemos podido comprobar en las JPA, a través de visitantes que reconocen que el propósito de su visita es "trobar-me amb la natura tranquil-lament".

Como señalábamos anteriormente, en el caso valenciano los yacimientos ibéricos visitables se ubican sobre todo en el interior de la provincia de Valencia. Se enmarcan, por tanto, en un pai-

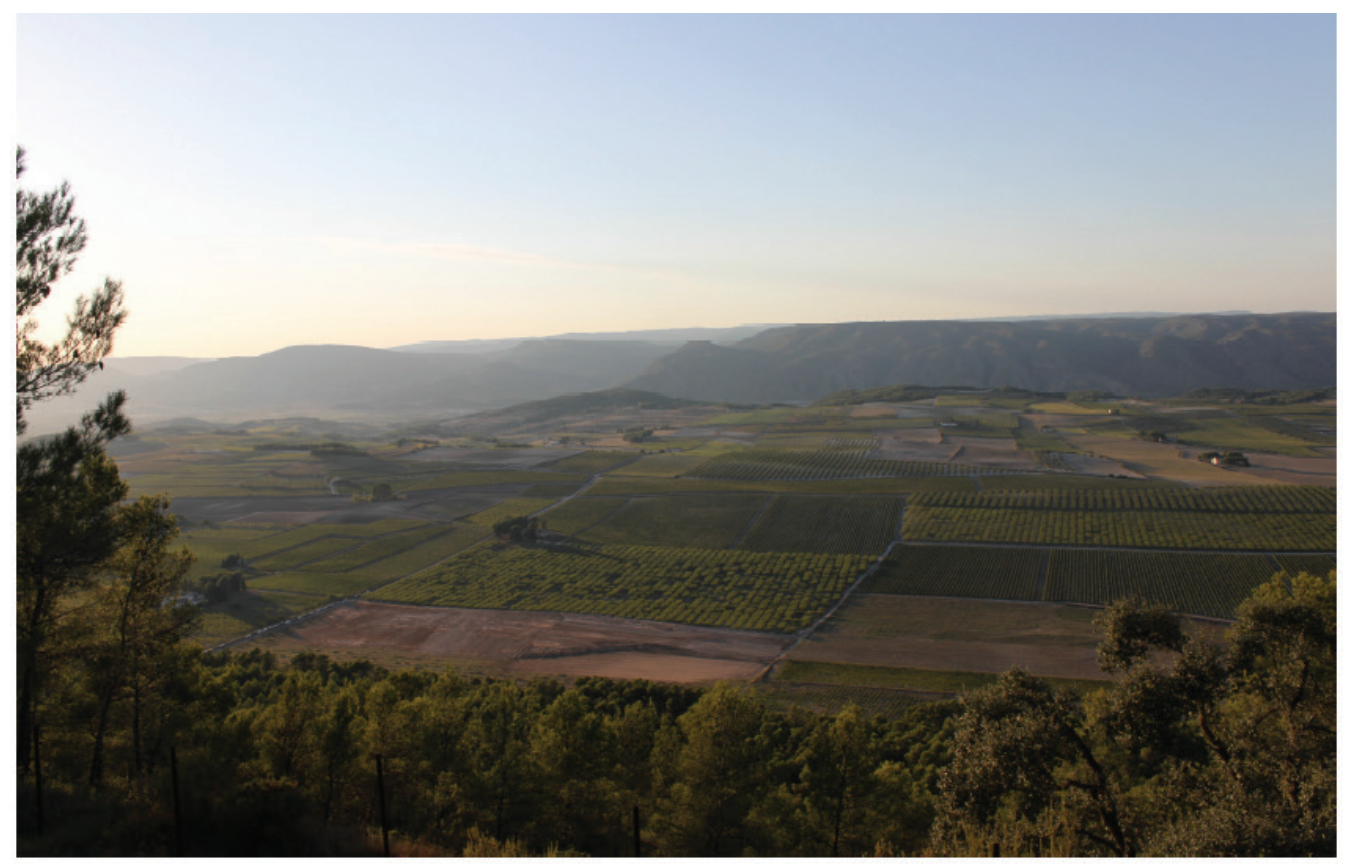

Figura 2. Vistas desde La Bastida de les Alcusses (Foto: A. Vizcaíno). 
saje caracterizado por su perfil montañoso, por el cultivo de cereales, viña y olivos, por la escasa ocupación demográfica y, en algunos casos, por las grandes masas boscosas (Fig. 2). Un entorno que encajaría con la noción de lo rural, que en el imaginario colectivo converge con la naturaleza y lo salvaje (Frigolé 2007).

Lo reseñable aquí es que esa imagen de ruralidad y naturaleza "salvaje" tiene su reflejo en la propia percepción social de los iberos, que suelen ser imaginados como un pueblo que habita en las montañas y sobrevive a través de actividades primarias. En efecto, del pasado ibérico se resalta un claro componente primitivista, que es herencia de la visión transmitida por las fuentes clásicas y de una concepción de la historia basada en el progreso. Algunos visitantes hablan de los iberos como "sobrevivents", "persona con imagen ruda y fuerte" o "culturas de subsistencia", y los ubican en poblados fortificados en altura. Los hay, incluso, que directamente asocian la cultura ibérica a conceptos como "rural", "subsistencia", "montañas", "la serra" o "altura". En cierto modo, al poner en relación el pasado ibérico con un paisaje de interior, montañoso y salvaje, se está contribuyendo a reforzar un imaginario particular en el que las nociones de subdesarrollo y primitivismo están muy presentes.

En cuanto a los museos, la ausencia de un paisaje que funcione como referencia -a excepción de los museos de sitio- se suple con la recreación de escenas de la época y con la alusión explícita a través de los textos. Ahora bien, del total de museos analizados muy pocos representan o hacen referencia al paisaje antiguo en sus discursos. El MARQ, el Museo Arqueológico de Guardamar del Segura (MAG), el Museo Arqueológico y de Historia de Elche (MAHE) y el Museo Histórico de Monforte del Cid (IBERO), son los únicos que introducen ilustraciones sobre el paisaje, que además, y debido a la ubicación de la mayoría de estos museos en la costa sur alicantina, representan zonas litorales con predominio de maquia mediterránea. Así, el visitante probablemente no encuentre diferencias significativas con el paisaje actual, por lo que se transmite la idea de continuidad. Incluso en algunos casos, como en el Museo Municipal de Requena, se habla de manera explícita de "un paisaje muy similar al actual".

La excepción más significativa a la hora de trazar continuidades paisajísticas entre pasado y presente la constituye el MAHE. En él, las numerosas ilustraciones que acompañan el recorrido expositivo no evocan un paisaje tan reconocible y de valor simbólico tan potente como es el Palmeral, definitorio de la imagen actual del yacimiento de L'Alcúdia -y utilizado en publicaciones divulgativas locales sobre los iberos-, sino que se representa un paisaje profundamente deforestado por la actividad agrícola. Pero lo que no se da en éste ni en ningún otro museo es la justificación de la elección del tipo de paisaje.

Así pues, el paisaje, sea real o recreado, no solo desempeña un importante papel en la evocación del pasado, sino también en el establecimiento de continuidades con el presente, con todo lo que ello implica desde un punto de vista identitario.

\subsection{Los restos arqueológicos}

Entrar en contacto físico y/o simbólico con la materialidad del pasado constituye el principal reclamo de las visitas tanto a yacimientos como a museos. Sin embargo, y a pesar de los puntos en común, la realidad de unos y otros difiere. Mientras los museos descontextualizan los objetos arqueológicos de su lugar de origen y los recontextualizan a través de discursos históricos y museográficos, los yacimientos muestran los restos -básicamente estructuras constructivas- in situ, acompañándolos también de discursos históricos pero sin los objetos que permiten reconstruir la vida en el pasado. Una situación que ocasionalmente genera descontento entre los visitantes al "no vore restes als jaciments. No deurien estar al museu". Por tanto, ya de partida existen diferencias en las capacidades explicativas y evocativas de museos y yacimientos.

Dejando a un lado el tema de la accesibilidad y la visibilidad de los restos arqueológicos, que como ya hemos señalado requeriría de un estudio específico, hay dos cuestiones fundamentales que influyen de manera directa en la percepción del pasado: la selección de épocas y la tipología de los restos exhibidos.

Respecto al primero, en general los museos arqueológicos ofrecen una visión diacrónica de la historia de los municipios y su territorio. Ésta suele abarcar desde la prehistoria hasta el final de la época antigua o, en su caso, hasta la Edad Media, siendo muy pocos los que incluyen tiempos más recientes. En el caso que nos ocupa, la totalidad de museos analizados contienen explicaciones sobre los iberos, pero lo interesante es detectar si dentro del espacio expositivo reciben mayor o menor atención que otras culturas. Calibrar esas diferencias proporciona algunos indicios sobre el pasado en el que buscan reconocerse los munici- 
pios, pues la selección de pasados no está motivada exclusivamente por la mayor abundancia de restos de una época u otra. También es resultado de un proceso de selección en el que entran en juego cuestiones como la monumentalidad y la singularidad, que favorecen una identificación mucho más efectiva con los visitantes.

No es casual, por ejemplo, que los museos que otorgan mayor protagonismo a la cultura ibérica sean el IBERO, que, además de su elocuente nombre, dedica casi todo su espacio a iberos y romanos; el MAHE, que en su recorrido desde la prehistoria hasta la época moderna utiliza una sala para cada periodo, excepto en el caso ibérico, al que dedica dos; o el MAG, en el que las salas de mayor tamaño se reservan al mundo fenicio e ibérico. Decimos que no es casual porque estos museos albergan las colecciones más ricas de la estatuaria ibérica del territorio valenciano, y no debemos olvidar que el arte de gran formato ha sido el que tradicionalmente ha marcado la valoración de la complejidad de las culturas antiguas (Aranegui 2012: 243). Así, poseer una plástica rica y monumental se convierte en motivo de orgullo y, por consiguiente, facilita la identificación de los visitantes en general y de los locales en particular. Todavía más, los tres museos mencionados cuentan entre sus colecciones con piezas que se han convertido en icónicas, aunque con desigual popularidad: la (réplica de) Dama de Elche en el MAHE, la (réplica de) Dama de Guardamar en el MAG y el toro del IBERO.

En otros casos, la centralidad del pasado ibérico en el museo no se da por una pieza singular, sino como reivindicación de su importancia dentro de la historia del territorio. Así ocurre en el Museu de Prehistòria de València, en el que la cultura ibérica asume el máximo protagonismo mientras otras, como la romana, ocupan un lugar secundario. Tal y como se ha señalado anteriormente, ese interés de Valencia capital por resaltar el pasado ibérico es resultado de un proceso de larga tradición afianzado a lo largo del s. XX. Desde Valencia se ha pretendido mostrar el pasado ibérico como rasgo singular del conjunto del territorio valenciano, del que actúa como motor identitario, y en especial de la provincia de Valencia. Así se ha hecho desde la esfera política, utilizando a los iberos como argumento identitario (Vizcaíno Estevan e. p. a), pero también desde instituciones culturales. En este sentido, el $\mathrm{Mu}-$ seu de Prehistòria de València, aún y siendo de titularidad y gestión provincial, ha tenido desde su fundación una clara vocación regional (Enguix y
Hernández 2006: 17) que puede rastrearse fácilmente en sus discursos.

Lo habitual en el resto de museos, sin embargo, es que haya un cierto equilibro en la representación de las distintas épocas. Evidentemente ese equilibro no debe entenderse en términos cronológicos, pues la prehistoria siempre aparece infrarrepresentada, sino como resultado de una división en periodos culturales definidos convencionalmente para estructurar la explicación.

En los yacimientos la lógica de la selección resulta algo más dramática desde el punto de vista patrimonial. Ya no hablamos de dedicar mayor o menor espacio a una cultura, sino de la eliminación de estructuras como parte del proceso de excavación y del acondicionamiento para la visita. Así, en los yacimientos se plantea la disyuntiva entre tratar de conservar y exhibir restos de fases y cronologías distintas, con las consiguientes dificultades de interpretación y comprensión para los visitantes; o privilegiar una época en concreto, ocultando o eliminando el resto, lo cual no siempre responde a criterios estrictamente científicos. En este sentido, la selección favorece una identificación más potente del territorio con ese pasado -en especial si la práctica se reitera- $y$, a la inversa, los pasados predilectos acaban estableciendo prioridades a la hora de intervenir sobre el patrimonio. Más incluso que en los museos, donde, independientemente del mayor o menor espacio concedido, se exhiben colecciones de casi todas las épocas.

Dejando de lado los yacimientos de ocupación exclusivamente ibérica, como La Bastida de les Alcusses, El Castellet de Bernabé (Llíria) o La Seña (Villar del Arzobispo), en la muestra estudiada encontramos dos comportamientos distintos. Por un lado, están aquellos que centran su foco de atención en el pasado ibérico y marginan otras épocas, como El Tossal de Sant Miquel (Llíria) y El Puntal dels Llops (Olocau). En ellos, a pesar de que las investigaciones han documentado fases de la Edad del Bronce (Bonet 1995; Bonet y Mata 2002), ni los paneles ni los folletos informativos las mencionan. Sean más o menos trascendentales desde el punto de vista científico, y dependa o no de otras cuestiones ajenas a la investigación, no deja de ser llamativo, sobre todo si tenemos en cuenta que se trata de dos de los yacimientos más emblemáticos del núcleo ibérico edetano.

Por otro lado están los yacimientos que incluyen estructuras de épocas distintas con explicaciones particularizadas. Así ocurre en La Illeta 
dels Banyets (El Campello), donde se exhiben estructuras desde el Neolítico hasta la época romana, y en El Tossal de Manises-Lucentum, donde el recorrido permite reconocer restos ibéricos y romanos. Pero quizá el caso paradigmático sea L'Alcúdia de Elche, en el que coexisten lo ibérico, lo romano y lo visigodo, y cuyo centro de interpretación construye un discurso transversal. Existen, no obstante, soluciones intermedias, como la de El Puig de la Nau (Benicarló), donde los restos visibles son de un momento muy concreto, el s. V a. C., pero se especifica que la ocupación del yacimiento comienza en la Edad del Bronce.

La segunda de las cuestiones que influyen en la manera de percibir el pasado a través de los restos arqueológicos es la tipología de yacimientos exhibidos. En el caso ibérico ha habido una predilección por los oppida, que es consecuencia de la importancia que se les ha otorgado en la investigación, pero también de su mayor extensión y monumentalidad, al menos en comparación con otros tipos de yacimiento. En la muestra analizada existe una diversidad relativa, pues aparte de los grandes centros (El Tossal de Sant Miquel, Los Villares-Kelin, L'Alcúdia, El Puig de la Nau, La Carència de Torís, La Bastida de les Alcusses) también son visitables algunos caseríos (El Castellet de Bernabé, La Seña), fortines (El Puntal dels Llops) e incluso pequeños emporios comerciales (La Illeta dels Banyets). Aún así, hay un predomino absoluto de los yacimientos relacionados con el hábitat. Solamente La Solana de Las Pilillas y la Rambla de la Alcantarilla de Requena, no incluidos en la muestra por no disponer de recursos explicativos, ofrecen una perspectiva distinta, pues se trata de estructuras de transformación de alimentos fuera de los asentamientos. Lo que no existen son necrópolis visitables, a pesar de que se hayan excavado algunas de carácter monumental; ni cuevas-santuario, ni otro tipo de infraestructuras como, por ejemplo, los caminos. Es cierto que las cuevas cuentan con la dificultad añadida de la accesibilidad y la presentación, ya que la ausencia de restos y estructuras in situ dificulta la percepción, como ocurre con los yacimientos paleolíticos (Ruiz Zapatero 2013). Sin embargo, la inclusión de estas otras tipologías de yacimientos permitiría diversificar y enriquecer la visión sobre la cultura ibérica, quizá excesivamente focalizada en los asentamientos de mayor rango.

En el caso de los museos, la mayoría exhiben un repertorio de objetos diversificado para ilustrar las distintas facetas de la vida cotidiana en época ibérica. En general el protagonismo recae en las piezas relacionadas con actividades productivas, si bien pueden rastrearse algunos comportamientos diferenciados. Por ejemplo, en el MAHE y en el IBERO, precisamente por la exclusividad que otorga del arte de gran formato, se ha hecho una apuesta clara por el mundo funerario. En cambio, la organización social, política y otras facetas muy recurrentes en otros formatos divulgativos, como la guerra, parecen tener un protagonismo menor.

\subsection{Los recursos informativos}

Los apartados vistos hasta el momento se refieren a factores que influyen de manera directa, aunque no siempre consciente, en la concepción del pasado ibérico. Sin embargo, son los recursos explicativos de museos y yacimientos los que verdaderamente construyen las narrativas históricas sobre el pasado y los que modelan de forma mucho más explícita esa imagen, de ahí que convenga prestarles mayor atención.

El discurso oficial se transmite en estos espacios a través de recursos variados, que van desde los medios impresos (folletos, guías, paneles, cartelas), pasando por los audiovisuales (audioguías, guías para stmarphones y tablets) hasta los que implican una intervención directa de los profesionales (visitas guiadas, talleres, jornadas de puertas abiertas, recreaciones históricas).

A través de esos recursos se van introduciendo los temas fundamentales para conocer a los iberos. Uno de los que más atención recibe es el del origen y la formación de la cultura ibérica. En general museos y yacimientos inciden en la idea de un proceso complejo en el que intervienen las poblaciones autóctonas y el componente griego y fenicio. Pero en algunos casos se hace todavía evidente la herencia de teorías colonialistas, al utilizar términos como "indígenas" frente a "colonizadores", o "comunidades" frente a "civilizaciones". Así, el contacto se entiende en clave de desigualdad cultural, donde los pueblos griegos y fenicios aparecen como motores casi exclusivos del cambio. En el Museu del Castell d'Onda se afirma que los colonizadores abrieron "un proceso irreversible de acultación [sic] sobre la población indígena"; en el Museu Municipal de Cullera se dice que los indígenas "evolucionaron a lo que se ha denominado Cultura ibérica, gracias a los contactos e influencias de los griegos y fenicios"; y en el Museu Arqueològic 
d'Oliva se reconoce "un procés d'aculturació del substrat indígena fins donar lloc, en el segle VI a. C., a la denominada Cultura Ibèrica". Es decir, se sobreentiende una unidireccionalidad, no un diálogo cultural. Hay algunas excepciones a este respecto, y por ejemplo en el MAHE se habla de una relación "beneficiosa para ambas partes" y en el MAG se resalta un intercambio en doble sentido.

La desigualdad cultural inicial, manifestada entre unos anónimos "pueblos indígenas" y unos griegos y fenicios más desarrollados, se convierte en complejidad cultural al tratar la cultura ibérica propiamente dicha. De hecho, los museos y los yacimientos son los únicos formatos divulgativos en los que siempre se incide en el desarrollo cultural de los iberos, a diferencia de lo que ocurre con muchos libros, novelas, cómics y documentales. El Museu de Prehistòria de València, por citar un caso evidente, habla en sus paneles de una cultura "equiparable por sus elementos distintivos al resto de civilizaciones mediterráneas".

La consideración de la complejidad cultural de los iberos cala, hasta cierto punto, en los visitantes. Así, algunos los ven como "los antiguos habitantes de nuestra región con una cultura y civilización avanzada", un "pueblo pre-romano, con una cultura muy avanzada" o un "pueblo que ha dejado mucho legado cultural e histórico"; incluso como un "mundo civilizado que es la base de nuestra cultura". Es más, a través del trabajo de campo etnográfico hemos podido comprobar cómo la explicación de elementos y prácticas culturales del mundo ibérico por parte de los guías, desafía, en muchos casos, la concepción primitivista con la que la gente llega a la visita. En La Bastida de les Alcusses, a los visitantes a menudo les sorprende que los iberos utilizasen llaves y tuviesen puertas en sus casas. Así lo reflejan opiniones como "es una cultura bastante desconocida, ¿no? Se conocen desde hace poco. Pero en realidad eran muy inteligentes", "iQué gente más lista por Dios!" o “iEran superdotados!”. La reconsideración de sus planteamientos previos les lleva a equiparar a los iberos a otras culturas supuestamente más desarrolladas, afirmando, por ejemplo, que "vivían como los romanos, los iberos estos". No obstante, y a pesar de los ejemplos citados, sigue existiendo una concepción primitivista dominante que lleva a concebirlos como "persones de l'època d'abans dels romans un poc primitius", "personages prehistoricos" o "casi prehistóricos".
La imagen primitiva de la cultura ibérica es en gran medida resultado del peso que continúa teniendo la idea del progreso en la concepción de la historia. En ella, el desarrollo se entiende en función de unos parámetros casi exclusivamente económicos y tecnológicos, de ahí que la antigüedad sea entendida como sinónimo de subdesarrollo cultural (Hernando 2005: 27-28). Esto explica que algunos visitantes perciban a los iberos como "la gente que vivía en esta zona y sobrevivía con la tecnología de la época". O que escolares de 8 años en un taller de tejido en el Museu de Prehistòria de València destaquen que "no tenían carros, iban en burros" o que "como no había ordenadores utilizaban máquinas de escribir", evocando, dentro de un imaginario infantil, binomios que contraponen grados de desarrollo tecnológico diferenciado.

Hasta cierto punto los museos y yacimientos contribuyen a perpetuar esa idea. Por un lado, ofrecen una visión lineal y teleológica de la historia caracterizada por la sucesión de etapas cronoculturales de carácter casi monolítico. Esa rigidez del pasado da poco margen a la diversidad y a los comportamientos diferenciados, y su fin último es argumentar la existencia de un presente que es la culminación del proceso. En el caso de los iberos, la diversidad suele limitarse a mencionar los nombres de las distintas regiones reseñadas en los textos clásicos, aunque algunos museos y yacimientos hablan de la ibérica como una cultura plural y diversa resultado de sustratos, influencias y procesos diferenciados.

Por otro lado, y en relación directa con la idea de linealidad y de progreso, está la centralidad que se concede al componente material y productivo en el discurso oficial. Condicionados por una manera tradicional de entender la arqueología, la mayoría de museos otorgan el papel protagonista a los objetos. Esto lleva muchas veces -e independientemente de que se recurra a museografías más o menos actualizadas- a reducir la explicación del pasado a una descripción de las tipologías y la utilidad de los objetos, casi siempre relacionados con la transformación de alimentos y con el comercio. En los yacimientos esta realidad se acentúa todavía más, ya que los paneles tienden a representar y describir las estructuras exhumadas, obviando la contextualización sociocultural.

Ocurre, al mismo tiempo, que el protagonismo otorgado a las estructuras y al componente productivo puede desembocar en una representación 


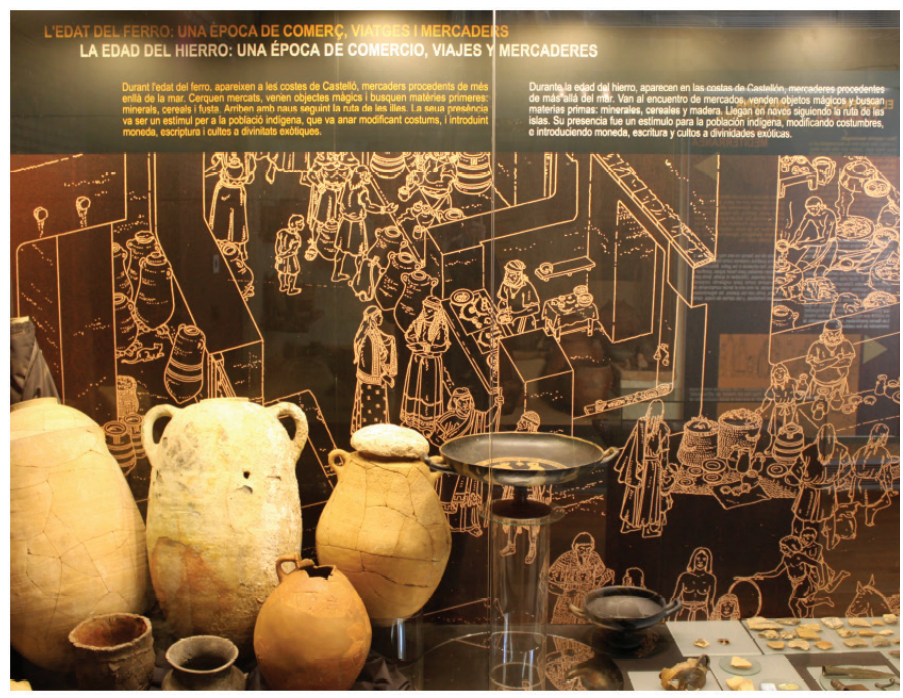

Figura 3. Escena de vida de un oppidum en el Museu de Belles Arts de Castelló (Foto: A. Vizcaíno).

deshumanizada del pasado. El repertorio visual de museos y yacimientos (ilustraciones, fotografías, recreaciones virtuales, reproducciones, maquetas) suele girar en torno a objetos y estructuras. En ellos, además, las personas o no aparecen o lo hacen como simple acompañamiento para especificar la escala y el funcionamiento de las piezas. La deshumanización puede darse también, paradójicamente, en las escenas en las que se recrea la vida de poblados y necrópolis. Aún así, este fenómeno es cada vez menos habitual (Mata 2014). De hecho, contamos con ejemplos interesantes de recreaciones (Fig. 3) que pueblan los espacios con personas, animales y objetos cotidianos que contribuyen a crear la imagen de un pasado menos frío y solemne.

Por otro lado, la ya comentada visión estática y homogénea de la cultura ibérica se traduce en la negación del cambio, al menos en aquellos ámbitos en los que pueda resultar más comprometido, como el de la organización social. Es cierto que la mayoría de museos y algunos yacimientos mencionan la jerarquización social. Incluso algunos, como el MARQ, el IBERO y el Museu Arqueològic d'Oliva, hipotetizan sobre la existencia de siervos y esclavos. Sin embargo, de lo que no se habla es del conflicto social, como si existiese una perfecta convivencia entre los distintos grupos y con las relaciones clientelares generadas. A pesar de que el conocimiento de este tema es limitado en el caso ibérico, la arqueología ha documentado, por ejemplo, evidencias de ensaña- miento con esculturas de damas y guerreros del sureste peninsular a finales del s. V a. C., lo cual habla de violencia y de transformaciones sociales (Zofío y Chapa 2005). Aún así, el IBERO es el único museo que menciona el conflicto, precisamente en relación con la destrucción iconoclasta. Es más, solamente unos pocos museos hablan de conflictos de alcance más amplio y de gran trascendencia para los iberos, como la Segunda Guerra Púnica.

Lo que se ofrece, pues, es una visión idealizada del pasado ibérico, definido por la centralidad de las actividades productivas, el desarrollo artístico y la estabilidad social. Una idealización que queda reforzada por el vínculo que se construye con lo mediterráneo -tan solo el IBERO habla de intercambios con el interior peninsular- que es entendido como lugar de cultura y de intercambio y cuna de la civilización. Consciente o inconscientemente, los discursos de los museos y yacimientos sancionan un orden social. Y esto es particularmente cierto para otra cuestión fundamental: la de los roles de género.

Las escenas representadas evidencian una masculinización de la cultura ibérica, que se manifiesta de tres maneras distintas. En primer lugar, por la infrarrepresentación de las mujeres en todos los escenarios. En segundo lugar, por la diferenciación entre actividades asociadas mayoritariamente a las mujeres, como las del ámbito simbólico-religioso o la propia inactividad, y a los hombres, sobre todo vinculados al ámbito 


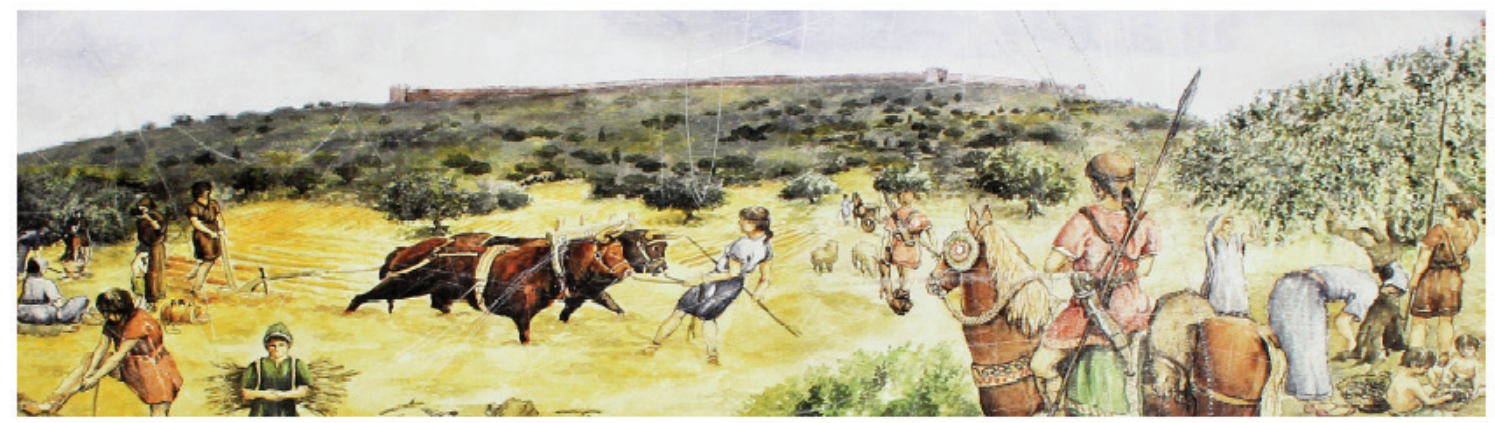

Figura 4. Hombres, mujeres y niños trabajando en una escena agrícola de un panel de La Bastida de les Alcusses (Foto: A. Vizcaíno).

productivo y bélico, aunque existen interesantes excepciones (Fig. 4). En tercer y último lugar, por la reiteración de la figura del guerrero como referente más reconocible de la cultura ibérica.

Esa proyección que se hace desde el presente, fundamentada más en clichés de nuestro tiempo que en evidencias arqueológicas, también afecta a otras cuestiones. Una de las más evidentes es la de trasvasar al pasado realidades político-territoriales actuales, lo cual acaba sesgando la concepción del mundo ibérico. En el caso de los museos, su ámbito oficial de actuación influye de manera decisiva. Los museos locales, que constituyen la mayor parte de la muestra, con frecuencia construyen un hilo conductor que se desentiende de las explicaciones globales y se centra en la escala inmediata, como si su historia pudiese plantearse al margen de procesos más amplios. Además, existe un interés manifiesto por encontrar referentes de prestigio (Ruiz Zapatero 2002) como el de los topónimos antiguos, aún y cuando las pruebas arqueológicas no sean concluyentes. Es el caso del Museu Municipal de Cullera, que utiliza la Sicana citada por los textos clásicos para el yacimiento de L'Alt del Fort, o el Museu del Mar de Santa Pola, que identifica La Picola con la antigua Alone.

A escala provincial, el Museu de Belles Arts de Castellón y el MARQ construyen un discurso muy ajustado a las fronteras. El caso alicantino es el más evidente, ya que casi circunscribe la antigua Contestania a la actual provincia de Alicante. Por su parte, el Museu de Prehistòria de València, aún y siendo de carácter provincial, construye un discurso autonómico y acopla el pasado ibérico a sus fronteras, hablando exclusivamente de ilercavones, contestanos y, sobre todo, edetanos. De hecho, en los mapas de este museo el territorio autonómico aparece desvinculado del resto del área definida arqueológicamente como ibérica.

En la asociación ibero-valenciana inciden otros museos, como el de Xàbia, donde se afirma que "las tierras valencianas [son] uno de los territorios en los que esta cultura presentará sus características más peculiares". Incluso en algunos casos se refuerza la identificación introduciendo en los textos de los paneles la primera persona del plural, como "nuestra zona", "nuestras tierras" o "nuestra geografia", y gentilicios actuales, como "nuestras tierras valencianas".

Estos planteamientos evidencian hasta qué punto el discurso autonomista ha modelado una manera de entender la historia. Es más, ese discurso tiene su reflejo en la concepción de parte de los asistentes a las JPA de La Bastida de les Alcusses y de Los Villares-Kelin. Un porcentaje nada desdeñable del total -entre el 10-15\%- ajusta la cultura ibérica a las fronteras del País Valenciano, incidiendo a menudo en su identificación como "primeros pobladores de la Comunidad Valenciana", "pueblos más antiguos de nuestra región" o "els primers".

Esa apropiación simbólica del pasado ibérico, que traduce un interés por hacer trascender la propia identidad, acaba generando desencuentros con quienes no comparten el discurso. Al fin y al cabo, la identidad implica diferenciación y conflicto. Esto explica, por ejemplo, que durante la escenificación de la vida cotidiana en las JPA de La Bastida de les Alcusses de 2012, visitantes procedentes de Madrid se mostraran contrarios a la explicación en valenciano, "ya que aunque financiado por la Diputación, la cultura y la historia no debe ser acaparada". La queja traduce una idea muy extendida y de larga tradición, según la cual lo ibérico debe entenderse en el marco de lo español. 


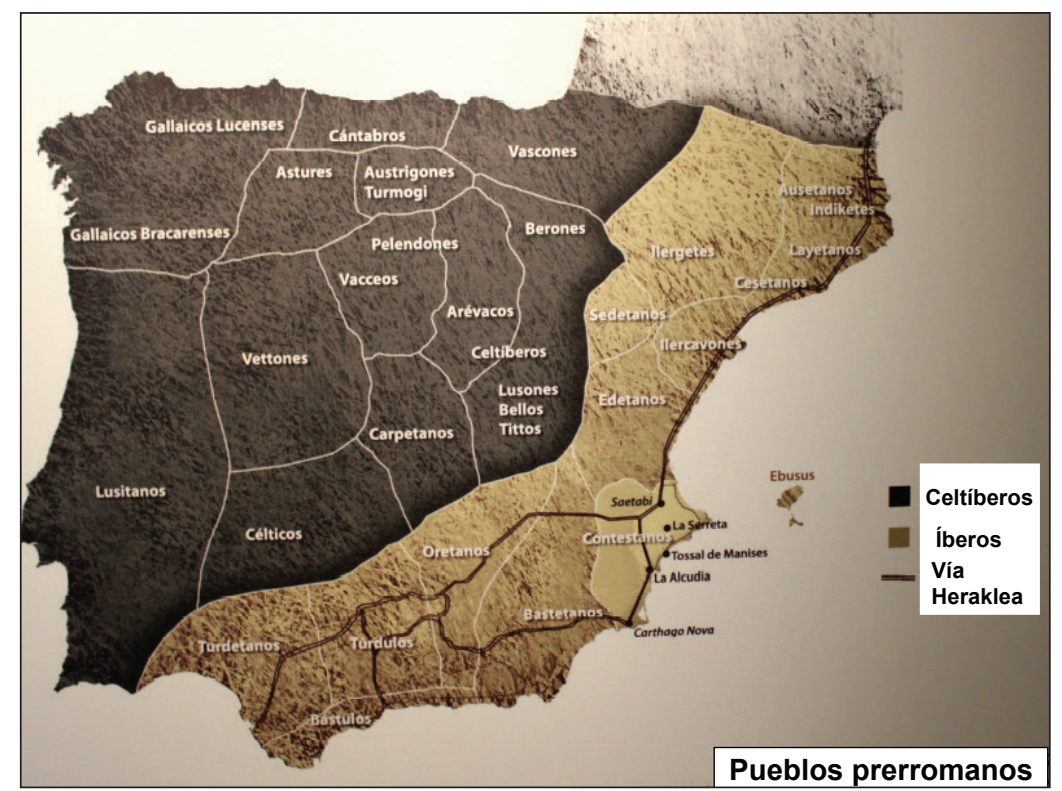

Figura 5. La cultura ibérica en un mapa del IBERO en el que se marca una clara frontera con Francia (Foto: A. Vizcaíno).

Los propios museos y yacimientos frecuentemente amoldan la cultura ibérica a España, levantando unas injustificadas fronteras con Francia (Fig. 5). Este tipo de recursos contribuyen a reforzar la identificación iberos-españoles que buena parte de la sociedad sigue asumiendo como válida, y que queda ejemplificada a través de las inquietudes de algunos visitantes, que llegan a preguntar "entonces eran españoles, ¿no?".

En esta misma línea, cuando en las JPA nos interesábamos por la primera imagen asociada a los iberos, algunos de los encuestados mencionaban España y la Península Ibérica. Incluso, aunque de manera muy puntual, hubo quien los identificó con Numancia y Viriato, evidenciando la todavía palpitante herencia de la educación franquista (Gil González 2012; Jimeno Martínez y de la Torre Echávari 2005).

También ocurre, en ocasiones, que el vínculo establecido con el pasado supera lo territorial y se adentra en lo cultural a través del reconocimiento expreso de la similitud entre objetos y prácticas del pasado y del presente. El Centre d'Interpretació de L'Alcúdia (Elche), por ejemplo, comienza el discurso expositivo con dos fragmentos cerámicos que apelan directamente a la identidad ilicitana. En uno se representa una serie de figuras portando palmas, donde la conexión con el popular Domingo de Ramos resulta sencilla. En el otro aparece una palmera, referente ineludible del paisaje y la identidad lo- cal. Por su parte, en el MARQ se señala la similitud entre las herramientas ibéricas y las de la agricultura tradicional -una comparativa que implícitamente se reconoce en buena marte de los museos que combinan material arqueológico y etnológico- y en el MAG se menciona la relación de la Dama de Guardamar con la leyenda de La Encantà, un relato muy popular en el municipio que cuenta con una celebración anual de carácter festivo.

La singularidad de algunas de esas piezas arqueológicas ha acabado convirtiéndolas en referente de identificación colectiva. Los casos de Elche y Moixent, con la Dama y el Guerrer, son evidentes, $\mathrm{y}$ en los propios espacios patrimoniales se ha buscado afianzar el vínculo. Tanto en La Bastida de les Alcusses como en L'Alcúdia de Elche hay reproducciones a gran escala de las piezas. En el caso ilicitano, además, habría que añadir la monumentalización del lugar del hallazgo del busto (Fig. 6) como espacio prácticamente sacralizado, al que, incluso, se llegan a hacer romerías (Vizcaíno Estevan 2015). Todavía más, en las recreaciones históricas que se llevan a cabo en estos dos yacimientos se recurre a la personificación de los iconos. Al final, el pasado ibérico, a través de los iconos -"el nostre Guerrer"-, se convierte en la clave para "darse a conocer por la importancia de los hallazgos", "donar a coneixer l'antiguetat de Moixen" y sentir "orgullo de que hayan vivido en mi pueblo". 


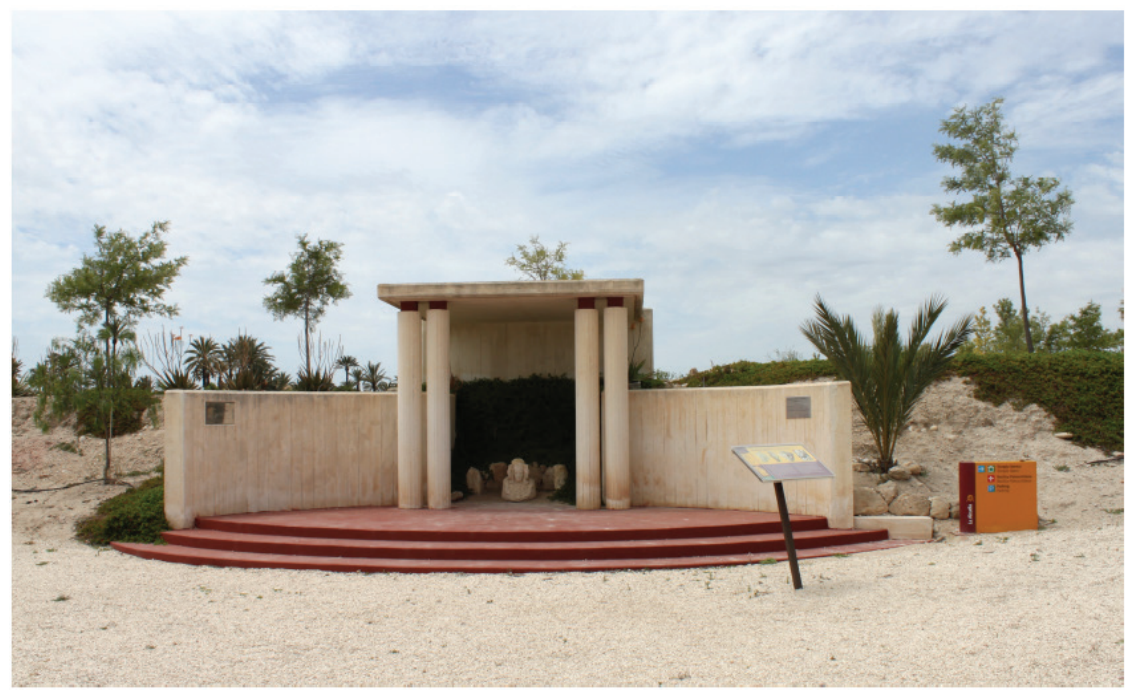

Figura 6. Monumentalización del lugar del hallazgo de la Dama de Elche en L'Alcúdia (Foto: A. Vizcaíno).

\section{Reflexiones finales}

El análisis de las narrativas en torno el pasado ibérico en los espacios patrimoniales nos conduce a lanzar una serie de reflexiones. La primera de ellas es que no podemos hablar de una realidad dicotómica entre los discursos oficiales y los de los visitantes. Muchas veces se concibe el discurso oficial como algo genérico, casi monolítico, quizá como una manera de reforzar la autoridad frente a las denominadas "visiones alternativas". Mientras tanto, las percepciones sociales se piensan como algo fragmentario y diverso, donde confluyen todo tipo de visiones. Sin negar la evidente y mayor heterogeneidad de los discursos emanados de los visitantes, conviene señalar que también en lo oficial existen disonancias y que la visión global dista de ser uniforme. Además, la supuesta equivalencia entre, por un lado, discurso oficial y veracidad y, por otro, percepciones sociales y distorsión, debe ponerse en entredicho. De igual manera que hay visitantes con una visión precisa del mundo ibérico y alejada de los estereotipos más comunes, ni los museos y ni los yacimientos están exentos de visiones ya no solo desfasadas -lo cual está condicionado en parte por las dificultades de actualización de los contenidos-, sino también claramente influenciadas por intereses que van más allá del conocimiento arqueológico, y que contribuyen a ofrecer una imagen distorsionada del pasado.

A lo largo del análisis se ha puesto de manifiesto que los discursos sobre lo ibérico, genera- dos tanto "desde arriba" como "desde abajo", están impregnados de un claro componente político e identitario. En efecto, la escenificación del pasado es también una escenificación sociopolítica referida al presente (Ederson 2002) que se manifiesta muchas veces de manera sutil. No es tan extraño que en los espacios patrimoniales se reitere, a través de las imágenes y los textos, la identificación del pasado ibérico con unos territorios concretos, o que se sancione un orden social en el que se manifiestan distintas formas de discriminación, entre ellas la de género.

Desde la arqueología conviene reflexionar y repensar este tipo de cuestiones, especialmente cuando se da por supuesto que museos y yacimientos son espacios privilegiados para el aprendizaje sobre el pasado, pero también sobre valores para la vida en el presente. Es cierto que buena parte de la sociedad llega a los museos y yacimientos con esos planteamientos ya aprendidos, como resultado de unos modelos educativos concretos -viejos y nuevos- y por los referentes que pueden encontrarse en los medios de comunicación. Pero no es menos cierto que si los museos y yacimientos reiteran esas realidades, los visitantes las identifican y las asumen como válidas por la autoridad que confieren esos espacios.

En cuanto a la conformación de la imagen de la cultura ibérica como cultura arqueológica, los discursos oficiales se esfuerzan en transmitir una idea de complejidad y desarrollo cultural. En general se huye de los estereotipos más recurrentes en los medios (Vizcaíno Estevan e. p. b), como la belico- 
sidad y el primitivismo, y se resalta la vida cotidiana frente a los grandes acontecimientos y las guerras. Es frecuente, en este sentido, que durante la visita a los sitios arqueológicos esos estereotipos se pongan en entredicho y tenga lugar una reconsideración del estatus de la cultura ibérica. En todo caso, el hecho de que la mayor parte de la sociedad siga teniendo una idea poco precisa y cuajada de clichés, no debería achacarse tanto a que los museos y yacimientos no cumplan con su misión, sino a que su impacto cuantitativo sea por regla general mucho menor al de otros formatos como, por ejemplo, la televisión.

Finalmente, es necesario llamar la atención sobre esos otros factores, analizados en este trabajo, que contribuyen a anclar ideas sobre el pasado ibérico en el imaginario colectivo. Unos factores que con frecuencia se dejan de lado al atribuírseles una cierta objetividad o al considerar que, simplemente, vienen dados. Nos referimos a la selección de épocas, al tipo de restos exhibidos, al paisaje y a la propia distribución de los espacios patrimoniales en el territorio.

En conjunto, todas estas variables nos permiten construir una perspectiva más amplia y compleja para comprender no solo qué imagen se está construyendo sobre la cultura ibérica como cultura arqueológica, sino también las funciones que ésta cumple en el presente.

\section{BIBLIOGRAFÍA}

Álvarez Vidaurre, E. (2011): Historia de la percepción del megalitismo en Navarra y Guipúzcoa. Aproximación a una biografía de sus monumentos. Universidad de Navarra, Pamplona.

Aranegui, C. (2012): Los iberos ayer y hoy. Arqueologías y culturas. Marcial Pons, Madrid.

ARANegui, C. (2015): Cuerpos sin rostro. Ostentación, violencia y representación social entre los iberos (siglos V-IV a. C.). Les estructures socials protohistòriques a la Gàl-lia i a Ibèria. Actes de la VII Reunió Internacional d'Arqueologia de Calafell (M. C. Belarte, D. Garcia y J. Sanmartí, eds.), Arqueomediterrània, 14: 23-37.

Ayán, X. (2015): Altamira vista por los españoles. JAS Arqueología, Madrid.

Azuar, R. (2013): Museos, arqueología, democracia y crisis. Trea, Gijón.

Ballard, S. (1997): Phenomenology, perception and the museum. Archaeological Review from Cambridge, 14(2): 93-104.

Bonet, H. (1995): El Tossal de Sant Miquel de Llíria: la antigua Edeta y su territorio. Museu de Prehistòria de València, Valencia.

Bonet, H.; Mata, C. (2002): El Puntal dels Llops. Un fortín edetano. Serie de Trabajos Varios del SIP, 99, Museu de Prehistòria de València, Valencia.

Bonet, H.; Ferrer, C.; Mata, C.; Vives-Ferrándiz, J. (2007): La Ruta Ibérica Valenciana. Una propuesta de desarrollo. IV Congreso Internacional sobre musealización de yacimientos arqueológicos. Conservación y presentación de yacimientos arqueológicos en el medio rural. Impacto social en el territorio, Santiago de Compostela: 337-343.

Collings, M. (2007): Roundhouses stories: reconstructions and public perceptions of the Iron Age. Which Past, Whose Future? Treatments of the Past at the Start of the 21st century. An international perspective (S. Grabow, D. Hull y E. Waterton, eds.), BAR Int. Series 1633, Oxford: 137-147.

Copeland, T. (2004): Presenting archaeology to the public: constructing insights on-site. Public Archaeo$\operatorname{logy}$ (N. Merriman, ed.), Routledge, London: 132-144.

Ederson, T. (2002): National identity, popular culture and everyday life. Berg, Oxford.

Enguix, R.; Hernández, M. (2006): El Servei d'Investigació Prehistòrica i l'Arqueologia Valenciana. Arqueologia en Blanc i Negre. La labor del SIP 1927-1950 (H. Bonet, M. J. De Pedro, C. Ferrer y A. Molina, eds.), Museu de Prehistòria de València, Valencia: 17-32.

Ferrer, C.; Vives-Ferrándiz, J. (2014): Patrimonio arqueológico y turismo. Unas reflexiones finales. El pasado en su lugar. Patrimonio, arqueología, desarrollo y turismo, Museu de Prehistòria de València, Valencia: 177-189.

Frigolé, J. (2007): Los modelos de lo rústico, lo salvaje y lo silvestre y la identidad de un valle del entorno del Cadí (Alt Urgell). Ecología política de los Pirineos. Estado, historia y paisaje (I. Vaccaro, O. Beltrán, eds.), Garsineu, Tremp: 157-171. 
Gil GonzÁlez, F. (2012): El uso de la figura de Viriato en la pedagogía franquista, Estudios de Historia de España, 14: 213-230.

Gustafsson, A.; KARLSSON, H. (2008): Places of power: control, public access and authenticity at rock carvings in Tanum, Sweden and Val Camonica, Italy. Public Archaelogy, 7(3): 174-198.

Hernando, A. (2005): Arqueología y Globalización. El problema de la definición del "otro" en la Postmodernidad. Complutum, 17: 221-234.

Hamilakis, Y.; Yalouri, E. (1999): Sacralising the past. Cults of archaeology in modern Greece. Archaeological dialogues, 2: 115-135.

Holtorf, C. (1997): Megaliths, Monumentality and Memory. Archaeological Review from Cambridge, 14(2): 45-66.

Holtorf, C. (2006): Experiencing Archaeology in Dream Society. Images, representations and heritage. Moving beyond modern approaches to Archaeology (I. Russell, ed.), Springer Science, Nueva York: 161-175.

Hornos, F.; RísQuez, C. (2005): Representación en la actualidad: las mujeres en los museos. Arqueología y Género (M. Sánchez Romero, coord.), Universidad de Granada, Granada: 479-490.

IbáÑez Alonso, M. (2014): Recepción y usos del patrimonio arqueológico de Sevilla. Universidad de Sevilla, Sevilla.

Jimeno Martínez, A. y de la Torre Échavarri (2005): Numancia. Simbolo e Historia. Madrid, Akal.

Lowenthal, D. (1998): El pasado es un país extraño. Akal, Madrid.

Mansilla, A. M. (2004): La divulgación del patrimonio arqueológico en Castilla y León: un análisis de los discursos. Tesis doctoral, Universidad Complutense de Madrid, Madrid [http://eprints.ucm.es/5133/]. Acceso el 29/5/2016.

Macdonald, S. (2005): Accessing audiences; visiting visitor books. Museums and Society, 3(3): 119-136.

Masriera, C. (2007): Anàlisi dels espais de presentació arqueològics de l'Edat dels Metalls. Tesis doctoral, Universitat de Barcelona, Barcelona.

Mata, C. (2014): De la naturaleza muerta al paisaje con figuras en la Arqueología Ibérica. Desmuntant Lara Croft. Dones, Arqueologia i Universitat (A. Vizcaíno, S. Machause, V. Albelda y C. Real, eds.), SAGVNTVM-PLAV, Extra 15: 43-48.

MECD (2015): Encuesta de Hábitos y Prácticas Culturales en España 2014-2015. Sintesis de resultados. Subdirección General de Estadística y Estudios, Ministerio de Educación, Cultura y Deporte, Madrid.

Merriman, N. (2000): Beyond the glass case. The past, the heritage and the public, UCL Institute of Archaeology Publications, Londres.

Moser, S. (2009): Archaeological representation: the consumption and creation of the past. The Oxford Handbook of Archaeology (B. Cunliffe, C. Gosden, R. A. Joyce, eds.), Oxford University Press, Oxford, 1048-1077.

Mouliou, M. (2008): Museum representations of the classical past in post-war Greece: a critical analysis. A singular Antiquity: Archaeology and Hellenic identity in twentieth-century Greece, Mouseio Benaki, Atenas: 83-109.

Pérez SAntos, E. (2008): El estado de la cuestión de los estudios de público en España. Mus-A, 10: 20-30.

PorTer, G. (1990): Gender bias: representations of work in history museums. The Australian Journal of Media \& Culture, 3(1): 70-83.

Querol, Ma A. (2014): Museos y mujeres: la desigualdad en Arqueología. Arqueoweb 15 [http://pendientedemigracion.ucm.es/info/arqueoweb/pdf/15/Querol15.pdf]. Acceso el 03/03/2016.

Querol, Ma A.; Hornos, F. (2011): La representación de las mujeres en los modernos museos arqueológicos: estudio de cinco casos. Revista Atlántica-Mediterránea, 13: 135-156.

Querol, Ma A.; Hornos, F. (2015): La representación de mujeres en el nuevo Museo Arqueológico Nacional: comenzando por la Prehistoria. Complutum 26(2): 231-238.

Rísquez, C.; Hornos, F. (2000): Paseando por un museo y buscando el lugar de la mujer. Arqueología Espacial, 22: 175-186. 
Rodríguez Temiño, I.; Ruiz Cecilia, J. I.; Mínguez García, C. (2015): Análisis de la visita pública a la Necrópolis Romana de Carmona entre 1885 y 1985. Archivo Español de Arqueología, 88: 263-282.

Ruiz ZAPATERO, G. (1998): Fragmentos del pasado: la presentación de sitios arqueológicos y la función social de la arqueología. II Seminari d'Arqueologia i Ensenyament (P. González-Marcén, ed.), Universitat Autònoma de Barcelona, Bellaterra: 7-33.

Ruiz Zapatero, G. (2002): Arqueología e identidad: la construcción de referentes de prestigio. Arqueoweb, 4(1) [http://pendientedemigracion.ucm.es/info/arqueoweb/pdf/4-1/ruizzapatero.pdf]. Acceso el 03/03/2016.

Ruiz Zapatero, G. (2013): Percibir, comprender y sentir. La accesibilidad de los sitios paleolíticos. Treballs d'Arqueologia, 19: 7-25.

Santamarina, B. (2008): Patrimonialización de la naturaleza en la Comunidad Valenciana. Espacios, ironías y contradicciones. Patrimonialización de la naturaleza. El marco social de las políticas ambientales. XI Congreso de Antropología de la FAAEE (O. Beltran, J. J. Pascual,I. Vaccaro, coords.), Ankulegi Antropologia Elkartea, Donostia: 27-44.

Scotт, M. (2005): "We grew up and moved on": Visitors to British Museums consider their "Cradle of Mankind". Envisioning the Past. Archaeology and the Image (S. Smiles y S. Moser, eds.), Blackwell Publishing, Malden: 29-50.

SмiтH, M. L. (2000): Archaeology, museums and the creation of national identity in the Indian subcontinent. The entangled past: integrating history and archaeology. Proceedings of the 30th Annual Chacmool Archaeological Conference (M. Boyd, J. C. Erwin y M. Hendrickson, eds.), University of Calgary, Calgary: 259-264.

Sommer, U. (2008): Some reflections on site presentation. Gestion et présentation des oppida. Un panorama européen. Management and presentation of oppida. A European overview, Glux-en-Glenne: 165-178.

Vizcaíno Estevan, T. (e. p. a): Roma no es suficiente. La invención del origen ibérico en el relato identitario de Valencia. Arkeogazte, 6.

Vizcaíno Estevan, T. (e. p. b): Los iberos vistos desde el sofá: el pasado ibérico en la cultura de masas. Actas del IV Congreso Internacional de Historia de la Arqueología. III Jornadas de Historiografía de la Arqueología SEHA-MAN, SEHA, Madrid.

Vizcaíno Estevan, T. (2015): De Damas a Vírgenes. La sacralización de algunos iconos ibéricos. Arqueologías sociales, arqueología en sociedad. Actas de las VII Jornadas de Jóvenes en Investigación Arqueológica, Arkeogazte, Vitoria: 209-217.

Zofío, S.; CHAPA, T. (2005): Enterrar el pasado: la destrucción del conjunto escultórico del Cerrillo Blanco de Porcuna (Jaén). Verdolay: Revista del Museo Arqueológico de Murcia, 9: 95-120 